\title{
MEMS scanners for display, imaging, and spectroscopy and their dynamic characterization
}

\author{
Hakan Urey", Huseyin R. Seren \\ Koç University, Electrical Engineering Department, Optical Microsystems Laboratory \\ Istanbul-Turkey
}

\begin{abstract}
Moving micro-mechanical structures combined with laser light sources and micro-optics enable a number of powerful applications in display, imaging, and spectroscopy. Examples of systems developed in our laboratory are: rotational scanners developed for micro-projectors, dynamic diffraction gratings with large out-of-plane motion developed for Fourier Transform spectrometers, and 2 degree-of-freedom MEMS stages that carry micro-lens arrays for laser beam steering and imaging applications. Precise control of motion is critical in all those applications. We developed a number of optical characterization tools for point-based and fullfield dynamic characterization of micro and nano mechanical structures. In this paper, we first briefly discuss the applications and then describe the details of the optical characterization tools. First setup is a stroboscopic interferometry for dynamic deformation analysis. Second setup is a simple technique for simultaneous in-plane and out-of-plane measurement with nanometric precision. The setup is constructed using one photo detector and a Mirau-type interference objective. For out-of-plane measurements, interference fringes are used to compute the the deflection amount. For in-plane measurements, knife edge technique is used to modulate the reflected beam intensity using a sharp edge in the object. Third setup is a simple optical angle sensor for rotational mirrors, which uses only one bi-cell photo detector. The setup is able to measure amplitude, phase, and quality factor of torsional devices.
\end{abstract}

Keywords: MEMS, actuators, imaging, display, MEMS characterization

\section{INTRODUCTION}

Micro-Electro-Mechanical-Systems (MEMS), or micro-systems, have been developed with an increasing demand in the markets for the last three decades. Microfabrication technologies increase the efficiency and make it possible to produce compact and low-cost devices. Integration of optics into these small structures finds a variety of applications which gradually becomes an indispensable need in the scientific world as well as in the daily life. In our research group, we have developed a number of optical micro-devices which are torsional mirrors for micro-projectors, high amplitude moving diffraction gratings for miniaturized spectroscopes, and 2D moving stages with micro-lens arrays mounted on them for large angle beam steering and imaging applications. There are various mechanical performance criteria for each device such as motion amplitude (deflection or rotation), dynamic deformation, or quality factor. Utilizing different optical techniques we developed a set of characterization methods for the mechanical performance measurements of the MEMS devices. Dynamic deformation is measured using stroboscopic interferometry which enables the measurement of the deformation at any specific position of the oscillation. For in-plane and out-of-plane moving devices a simple experimental setup is built to extract amplitude and frequency responses. A Mirau objective with a laser source is used to measure the out-of-plane motion amplitude again using interferometry techniques. With the same setup, in-plane motion

*hurey@ku.edu.tr; phone +90 212 338-1474; http://home.ku.edu.tr/ mems/

Fourth International Conference on Experimental Mechanics, edited by C. Quan, K. Qian, A. Asundi, F. S. Chau, Proc. of SPIE Vol. 7522, 75221P - (C) 2010 SPIE · CCC code: 0277-786X/10/\$18 · doi: 10.1117/12.851777 
amplitude is also measured by focusing the light on the edge of the moving platform and using so called knife edge technique. Another simple setup with a dedicated bi-cell photo detector circuitry is built to measure the rotation amplitude, phase and quality factor of torsional MEMS scanners.

\section{MEMS DEVICES}

\subsection{D MEMS stages}

Optical beam steering is a very common application field for micro-system technology. Numerous methods have been developed to reach high steering angles within a small form factor. Our group showed that scanning micro-lens arrays (MLA) are good candidates for such requirements [1]. Where imaging is needed within a constrained volume, e.g. endoscope or surgery probes, this method can be implemented as shown in the Figure 1.

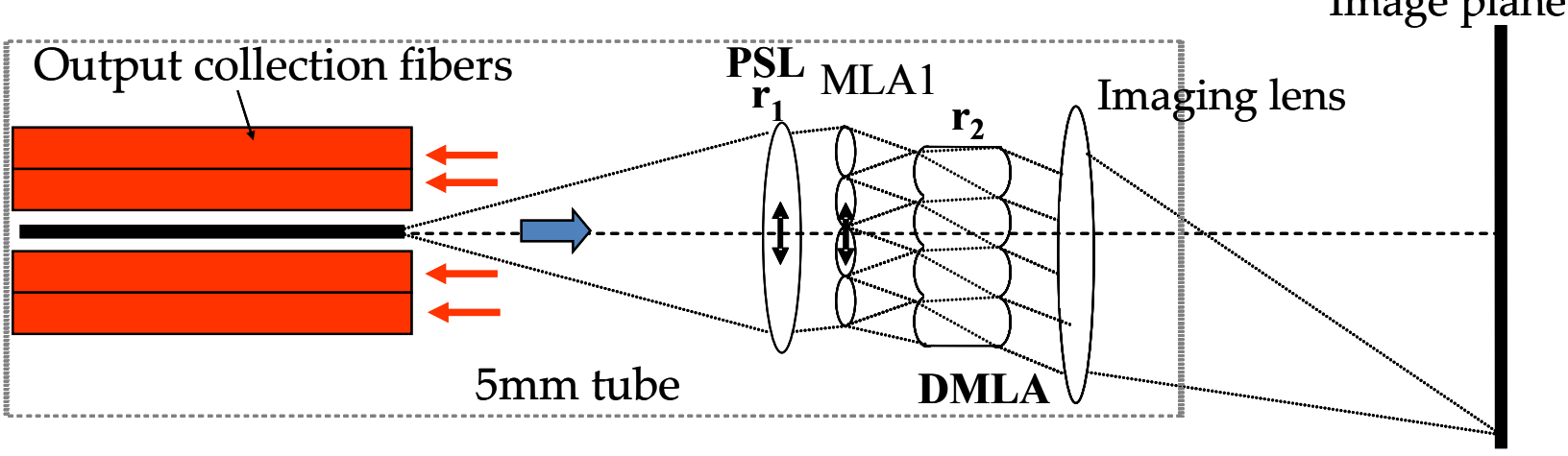

Figure 1. MLA scanning system

A MEMS stage which is able to move both in-plane and out-of-plane directions is developed to carry an MLA and perform a 2D scanning [2]. An MLA is fabricated and mounted on the stage. A prescan lens (PSL) which is also carried by another 2D MEMS stage is used to homogenize the scanning by preventing discrete addressing.

\subsection{MEMS device for FTIR lamellar grating interferometer based spectrometers}

Spectrometers are widely used instruments for qualitative and quantitative material detection and Fourier Transform Infrared (FTIR) spectroscopy is a well established method. Beyond the research laboratories, various industrial fields also need spectrometers for applications such as quality control, mine and bomb detection. However, FTIR spectrometers are mostly bulky and fragile instruments. Therefore, building smaller and sturdier FTIR spectrometers is needed. Lamellar grating interferometer based spectrometers can satisfy these needs with compact structure and reasonable alignment tolerances [3][4]. We developed an LGI MEMS device for high resolution miniaturized spectrometers in the course of EU funded MEMFIS project [www.memfis-project.eu] [5]. The device is composed of two comb sets, one is stationary and the other one is movable, a back bone that carries the movable comb set, and spring suspensions that are connected to the backbone (Figure 2). The performance of the device is highly depends on the out-of-plane motion amplitude and the flatness of the reflective surface during the operation. 


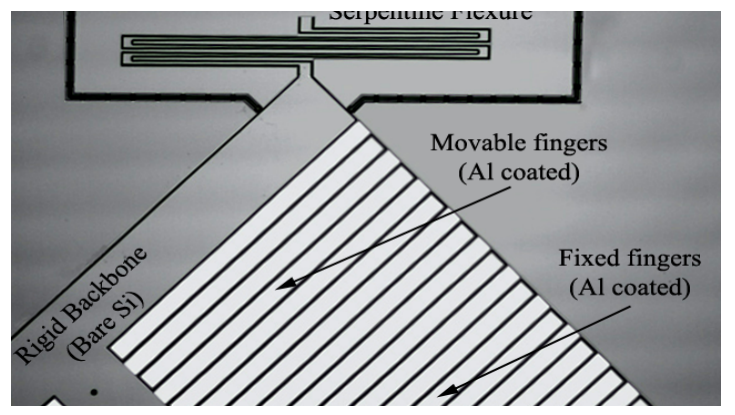

Figure 2. SEM picture of the MEMS device for Fourier transform spectrometer developed in our group illustrating fixed fingers and Silicon die, movable fingers, rigid backbone, and serpentine springs [3]

\subsection{Torsional scanners}

Similar to beam steering, beam scanning is also a major application field of MEMS technology. Barcode scanners, laser based displays, and laser printers are some of the well known applications where MEMS scanners play the key role. These applications require good control of the device during the operation. Therefore, the dynamic behavior of the device should be well known prior to operation so that the control system can be established for necessary addressing.

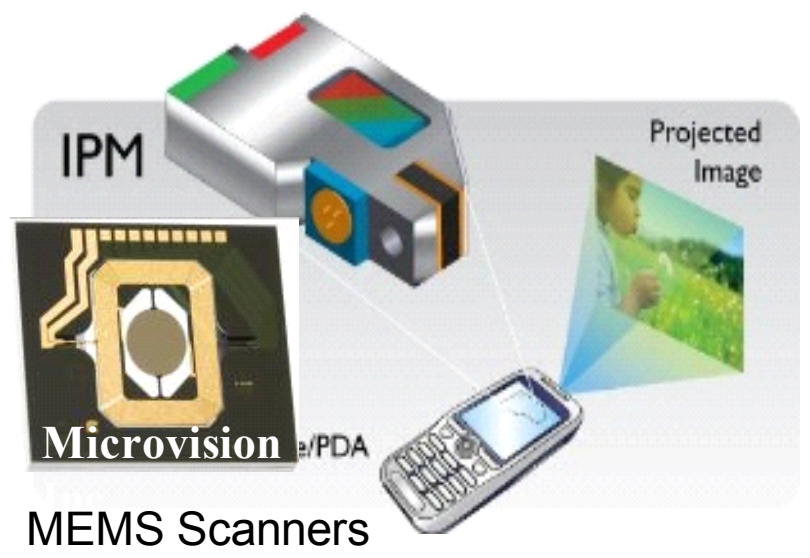

Figure 3. Torsional MEMS scanner designed for handheld projectors. Photo courtesy of Microvision Inc. (www.microvision.com)

Figure 3 illustrates a magnetic actuated 2D MEMS scanner developed for mobile projector applications. Our group is active in MEMS scanner development for many years and in collaboration with Microvision developed a highperformance electrostatic actuated MEMS torsional scanner for mobile-projection applications [6]. It is able to make high amplitude rotation for high resolution projection. The dynamic deformation of the mirror should be kept at minimum even at high amplitudes in order to maintain the display quality.

\section{CHARACTERIZATION METHODS}

\subsection{Stroboscopic interferometry}

Dynamic deformation of the reflective surfaces has a crucial effect on optical MEMS devices. In imaging and display applications, non-flat surfaces act like a curved mirror and reduce the image or display quality by changing focal plane of the optical system. In spectrometer applications, a curved reflective surface results in a noise in the collected interference data and a poorer spectral resolution.

Building a stroboscopic interferometry setup as shown in the Figure 4 the variation profile of a reflective surface can be 
monitored and measured [7]. A flat static mirror is used as a reference. The light is split into two using a beam splitter and reflections from static mirror and moving MEMS mirror is collected back on a CCD camera. The light is modulated synchronized with the moving mirror, i.e. at the same exact frequency. This creates a stationary fringe pattern on the CCD camera which looks like izohips curves. The deformation amount between successive curves corresponds to half of the wavelength. The camera output can be evaluated using a post-processing algorithm with sub-wavelength precision. By changing the phase difference between the light modulation input and MEMS device actuation input, the dynamic deformation at different positions of the oscillation can be observed.

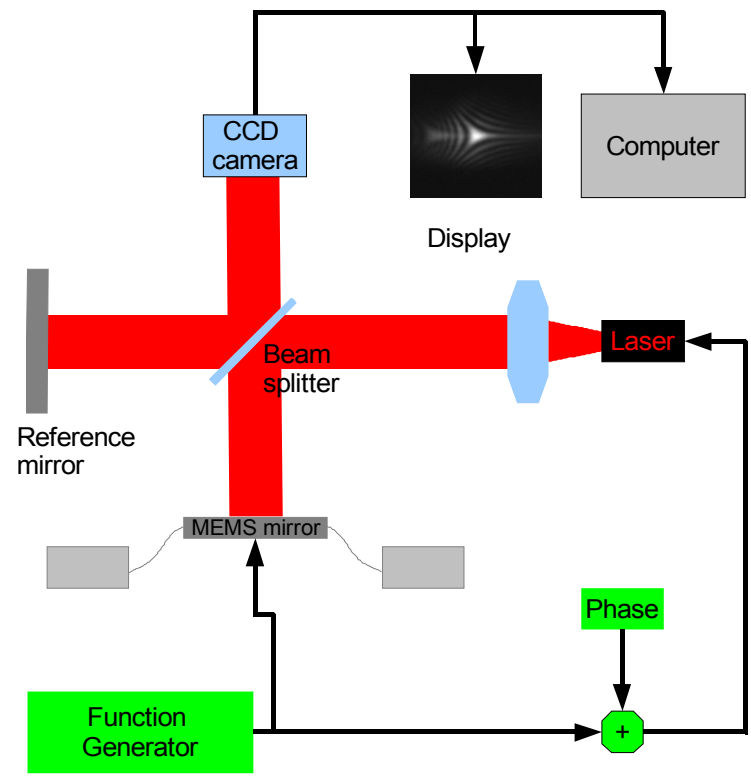

Figure 4. Stroboscopic interferometry setup for dynamic deformation
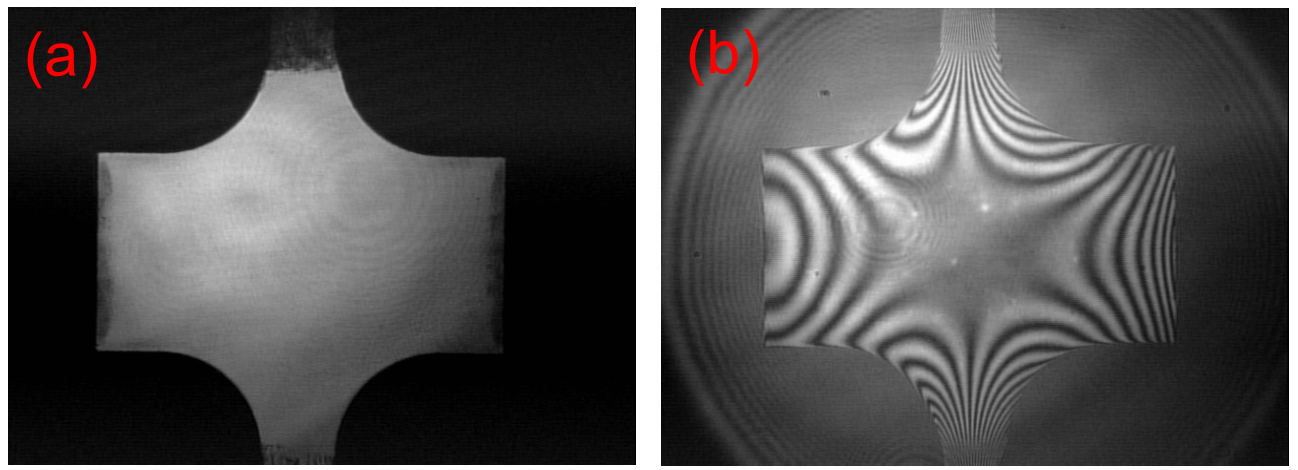

Figure 5. Dynamic interferometric images of a torsional micro-scanner illustrating the dynamic deformation at $17.5 \mathrm{kHz}$ and (a) at rest position (b) at full angle rotation

\subsection{D deflection characterization setup}

We developed a novel setup that can be used for the characterization of both in-plane and out-of-plane moving micro/nano devices [8]. An interference objective is used as a key element. Primary role of this objective is to be a reference mirror for interferometric out-of-plane measurement. Secondary purpose is to focus the light onto the edge of the sample for in-plane measurements with knife edge technique. The setup is able to provide high resolution with large dynamic range in both kinds of motions. 


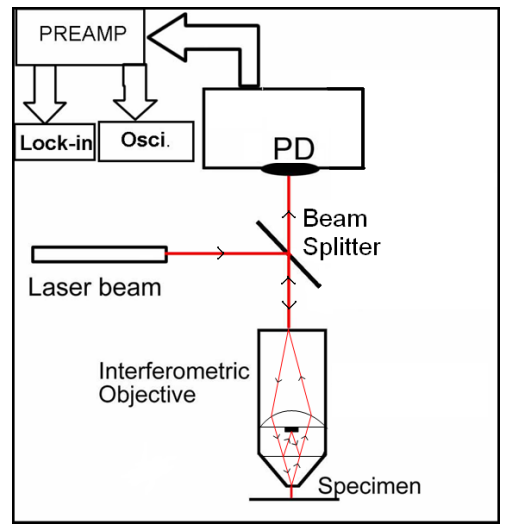

Figure 6. 2D deflection sensor setup

The reflected laser light from the out-of-plane moving device interferes with the reflected light form the objective's reference mirror. The output, interference intensity, is modulated as a function of the motion. The motion amplitude can be measured by counting the number of fringes if the motion is large, i.e. more than a wavelength. Generally devices exercise a sinusoidal oscillation which in return results as a chirp shaped output. If the motion amplitude is less than a quarter of the wavelength, the output is observed similar to a sine wave. A calibration measurement with a computer controlled precision stage is needed for precise and correct result. $4 \mathrm{pm} / \sqrt{\mathrm{Hz}}$ resolution is achieved with this method.
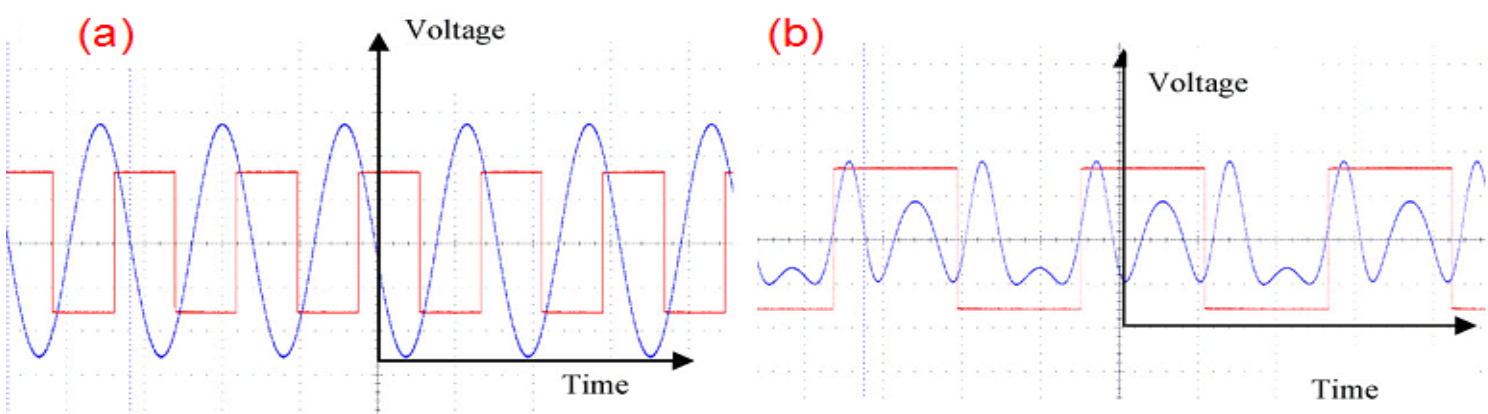

Figure 7.Output examples for (a) small (39nm) (b) large (906nm) out-of-plane motions. Square signals show the TTL output of the actuation input signal.

In-plane motion amplitude is measured using the knife edge technique. In this technique, the laser spot is focused on the edge of the moving part of the device and the modulation at the reflected light intensity is observed. The precision and the dynamic range of the measurement can be adjusted by playing with the spot size. The spot profile and size has to be calibrated using a precision stage prior to the experiment. We acquired $100 \mathrm{pm} / \sqrt{ } \mathrm{Hz}$ resolution for $1 \mu \mathrm{m}$ full width spot size at the half of the maximum intensity.
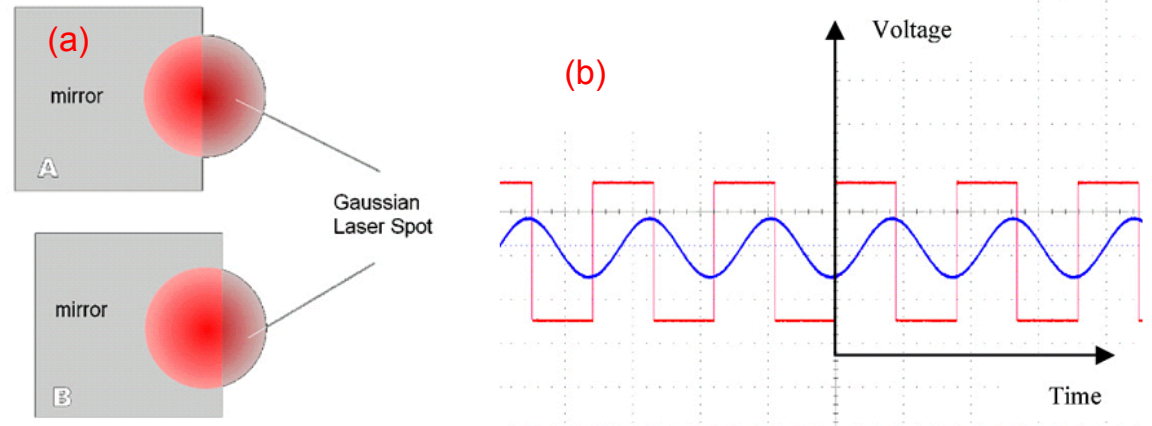

Figure 8. (a) The sketch showing the knife edge configuration (b) An examplary output for a 5um in-plane motion 


\subsection{Torsion characterization setup}

We built a single detector based torsional characterization setup [9]. Since in most of the applications dynamic feedback is needed, this method can also be implemented as a built in feedback system. The technique is based on a bi-cell photo detector which produces a trigger signal at each pass of a laser spot across the two parts of the detector. Therefore, by placing the detector at a foreknown position in the scan line, the oscillation amplitude, phase, and damping factor can be deduced by using a circuitry that converts the detector output into a square wave. The duty cycle and period of the resulting square wave carry the necessary information for the amplitude measurement.
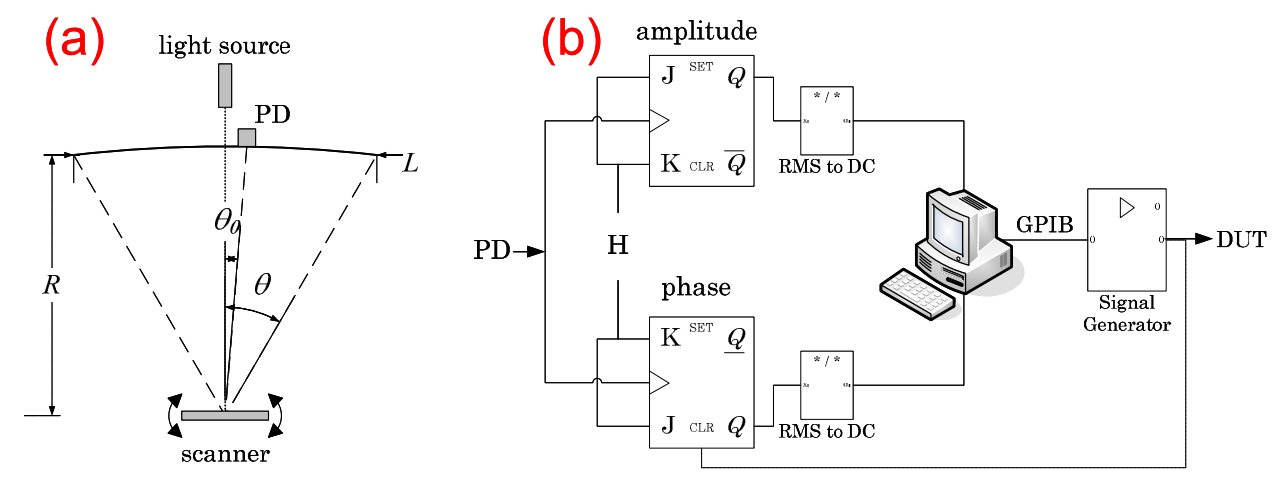

Figure 9. Torsional scanner characterization setup (PD stands for the photo detector) (b) read-out circuitry

By modulating the circuit (or flip-flop) synchronized with the actuation signal, the output can also be related to the phase difference between the input and the scanner. A sample measurement output is shown in the figure below.
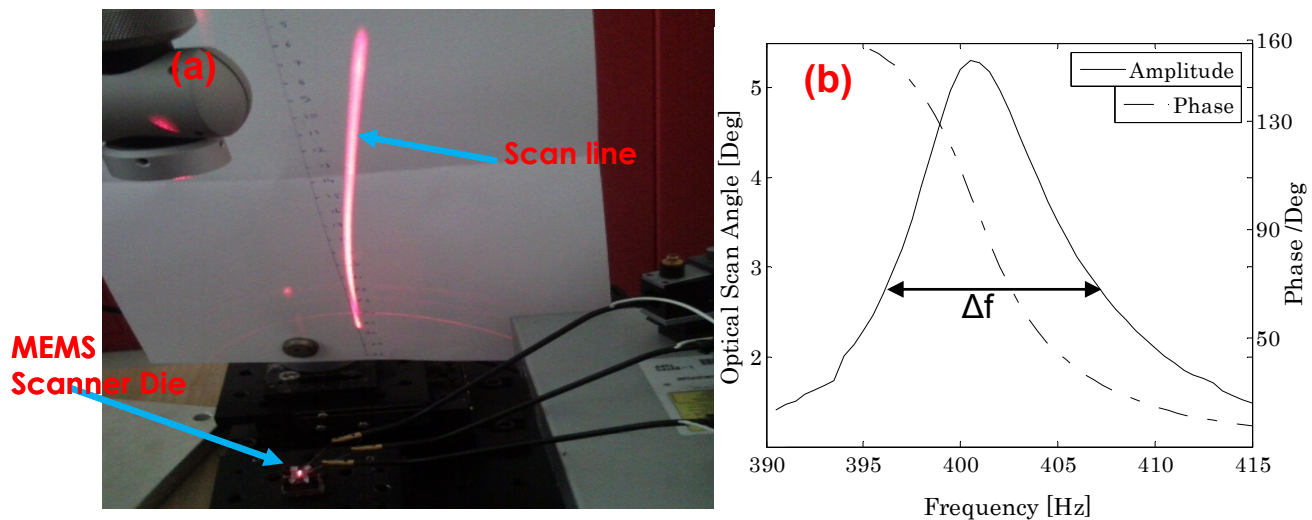

Figure 10. (a) A MEMS scanner and scan line, (b) Amplitude and phase frequency response of a torsional scanner $(\Delta \mathrm{f}$ is the bandwidth based on full width at half maximum)

The damping constant and the quality factor of the device can be calculated using the $3 \mathrm{~dB}$ bandwidth of a linear system (shown as $\Delta \mathrm{f}$ in the Figure 10.(b)). However, not every system are linear. It is also possible to measure the quality factor of the system by observing the decay of the oscillation when the actuation input is shut down. An example detector circuit output and corresponding decaying amplitude is shown in the

Figure 11. 


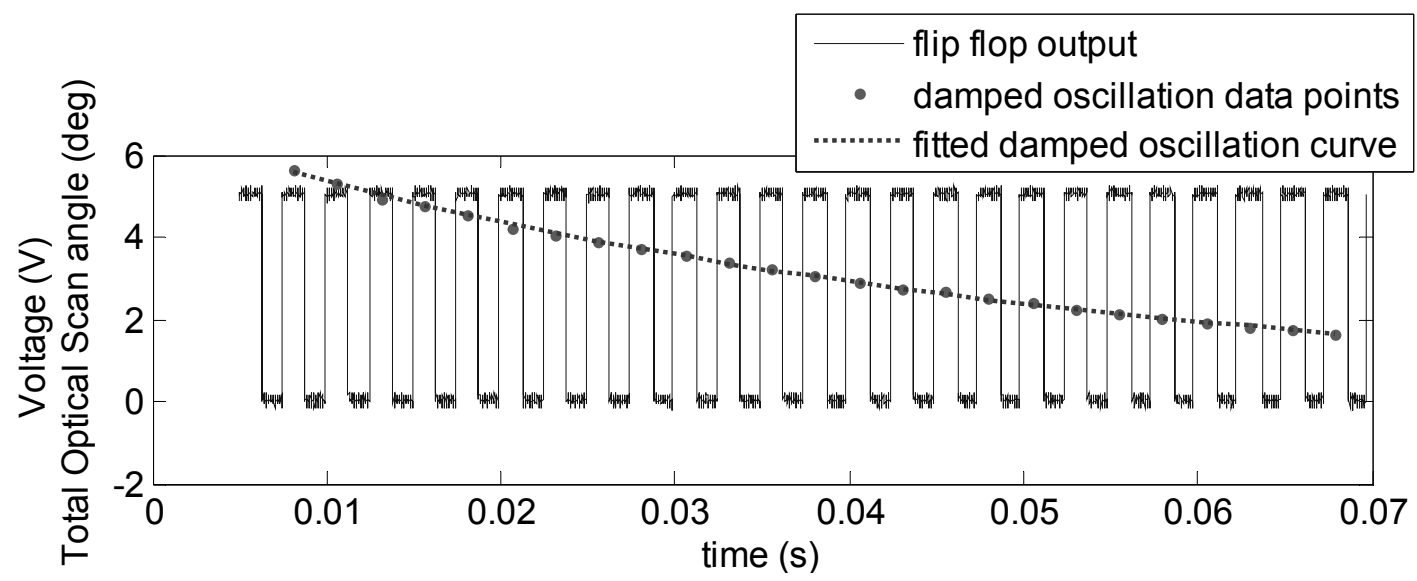

Figure 11. Decaying characteristics of a hysteretic micro-mirror. Square wave is the detection circuitry output, big dots are computed amplitudes at corresponding time intervals, and small dots are the fitted exponential function.

This easy to implement method's precision can be as good as 1/10000 depending on the detector position. As the detector placed closer to the end of the scan line, the precision becomes better related to the speed of the traveling laser spot.

\section{CONCLUSIONS}

Characterization of micro-systems carries a great importance for understanding their behaviour and advancing the research and development in this field. Optical applications constitutes a significant portion of the micro-systems and optical characterization methods are widely used. Optical measurement techniques provide high precision and robust results. In Optical Micro-systems Laboratory, we developed easy to implement optical and opto-electronical measurement techniques which enables us to characterize a variety of devices such as in-plane or out-of-plane moving, or rotational micro scanners.

\section{ACKNOWLEDGMENTS}

Partial supports from TUBITAK grant 106E068, Microvision Inc., USA, and FP7 MEMFIS project are gratefully acknowledged. H. Seren also thanks TUBITAK for Graduate Studies Scholarship Programme. H. Urey acknowledges TUBA-GEBIP award. Authors also thank Caglar Ataman, Aslihan Arslan, Sertan Kutal Gokce, Ata Akatay, and Selim Olcer for their contributions.

\section{REFERENCES}

[1] Akatay, A., Urey, H., "Design and optimization of microlens array based high resolution beam steering system",Optics Express, Vol. 15, No. 8, p. 4523-4529, 2007

[2] Gokce, S. K., Holmstrom, S., Hibert, C., Ataman, C., Arslan, A., Seren, H. R., Urey, H., "MEMS stage integrated with microlens arrays for high-resolution beam steering", Eurosensors XXIII, Pages 1319-1322, Lausanne, Switzerland, 2009

[3] Ataman, C., Urey, C., Wolter, A., "A Fourier transform spectrometer using resonant vertical comb actuators", Journal of Micromechanics and Microengineering, Vol. 16, p. 2517-2523, 2006.

[4] Manzardo, O., Michaely, R., Schadelin, F., Noell, W., Overstoltz, T., de Rooij, N. F., Herzig, H.P., "Miniature lamellar grating interferometer based on silicon technology", Optics Letters, Vol. 29, p. 1437-1439, 2004.

[5] Seren, H., "MEMS fourier transform spectrometer using lamellar grating", MS Thesis, Koç University, Istanbul, Turkey, 2009 
[6] Arslan, A., Ataman, C., Holmstrom, S., Hedsten, K., Enoksson, P., Seren, H. R., Urey, H., "Mechanically coupled comb drive MEMS stages", IEEE Optical MEMS and Nanophotonics, p. 140-141 Freiburg, Germany 2008

[7] Urey, H., Wine, D. W., Osborn, T. D., "Optical performance requirements for MEMS-scanner based microdisplays", Conf. on MOEMS and Miniaturized Systems, SPIE Vol. 4178, p. 176-185, 2000

[8] Seren, H., Urey, H., "Optical sensor for micro and nano mechanical system characterization", Eurosensors XXII, p. 1292-1295, Dresden, Germany, 2008

[9] Ataman, C., Seren, H. R., Schenk, H., Urey, H., "Dynamic characterization of MEMS scanners", Sensors and Transducers, Vol. 108, Issue 9, p. 31-39, September 2009" 